\title{
Predictors of associated and multiple autoimmunity in children and adolescents with type 1 diabetes mellitus
}

\author{
Konstantinos Kakleas', \\ Lydia Kossyva', \\ Anastasia Korona', \\ Nikolitsa Kafassi ${ }^{2}$, \\ Spyridon Karanasios ${ }^{1}$, \\ Kyriaki Karavanaki ${ }^{1}$
}

'Diabetic Clinic, 2nd Department of Pediatrics, National and Kapodistrian University of Athens, 'P. \& A. Kyriakou' Children's Hospital, Athens, Greece 'Department of Immunology, 'Laiko' General Hospital, Athens, Greece
Received: 13 August, 2021

Revised: 10 October, 2021

Accepted: 1 November, 2021

Address for correspondence:

Konstantinos Kakleas

Athens General Children's Hospital

"Pan. \& Aglaia Kyriakou", Thivon

kai Levadias, Athens P.C. 11527

Telephone: 0030-213 2009000

Email:koskakl2@yahoo.gr

https://orcid.org/0000-0002-79938418
Purpose: Type 1 diabetes mellitus (T1DM) is an autoimmune condition characterised by the presence of antipancreatic antibodies. The autoimmune process is also directed against other organs, most frequently against the thyroid gland, intestinal mucosa, and gastric parietal cells.

Methods: Our investigation included 121 children with T1DM with a mean age \pm standard deviation of $11.99 \pm 4.63$ years (range, $2.0-20.0$ years). We explored the frequency of associated autoimmunity; the presence of predictive factors such as current age, sex, and severity at diabetes diagnosis; T1DM duration; and family history of autoimmunity.

Results: Associated autoimmunity was present in $28.9 \%$ of T1DM patients. Children with associated autoimmunity were older at diabetes diagnosis $(P=0.009)$ and had a longer diabetes duration compared to children without associated autoimmunity $(P=0.044)$. Adolescents aged $12-20$ years had a statistically significant higher chance of developing thyroid autoimmunity compared to children aged 1-5 years $(P=0.019)$. Multiple autoimmunity (MA), T1DM, and 2 or more autoimmune diseases were present in $5.8 \%$ of the study population. All children with MA presented with ketoacidosis at diabetes diagnosis and had a higher percentage of familial autoimmunity $(P=0.042)$. The familial autoimmunity of these patients most frequently affected $\geq 3$ relatives $(P=0.026)$ and was more frequently diagnosed before 5 years of age ( $P=$ not significant).

Conclusion: Associated autoimmunity was present in almost one-third of T1DM patients. Significant associations with associated autoimmunity were longer diabetes duration, female sex, older age at diabetes diagnosis, and glutamic acid decarboxylase positivity. Predictors of MA were age $<5$ years at T1DM diagnosis, the presence of diabetic ketoacidosis at diagnosis, and a significant family history of autoimmunity.

Keywords: Type 1 diabetes mellitus, Child, Associated autoimmunity, Multiple autoimmunity, Predictors

\section{Highlights}

- Approximately one third of children and adolescents with type 1 diabetes mellitus (T1DM) had associated autoimmunity and 5.8\% multiple autoimmunity. Predictors of associated autoimmunity were older age, longer diabetes duration and female sex.

Children with multiple autoimmunity are more likely to be diagnosed with T1DM at an age younger than 5 years, to have DKA at diabetes diagnosis and a significant family history of autoimmunity.

It is important to regularly screen T1DM children and adolescents for associated 
autoimmunity, especially those with GADA positivity and a significant family history of autoimmunity, for the early identification and management of associated autoimmune diseases.

\section{Introduction}

Type 1 diabetes mellitus (T1DM) is an immune-mediated destruction of pancreatic beta-cells resulting in the loss of insulin production. ${ }^{1)}$ T1DM often coincides with other autoimmune diseases such as autoimmune thyroiditis (15\%$30 \%)$, celiac disease (CD) (4\%-9\%), autoimmune gastritis/ pernicious anaemia $(0.3 \%-5 \%)$, and Addison disease $(0.5 \%){ }^{2)}$ Less frequently T1DM coexists with rheumatoid arthritis, autoimmune hepatitis, vitiligo, alopecia, and psoriasis. This coexistence is often present in autoimmune polyendocrine syndromes I-III. ${ }^{3)}$ Various factors have been associated with multiple autoimmunity, such as genetic background, sex, age, age at diabetes diagnosis, diabetes duration, and family history of autoimmunity., ${ }^{4,5)}$

The aim of this study was to investigate the frequency of multiple autoimmunity, defined as the presence of more than one autoimmune disease in addition to diabetes, in children and adolescents with T1DM and to identify any predictive factors, such as the presence of pancreatic autoantibodies, current age, sex, diabetes duration, age at diabetes diagnosis, and family history of autoimmunity.

\section{Materials and methods}

\section{Study population}

The study included 121 children. Japanese Diabetes Society diagnostic criteria for acute onset T1DM were: (1) occurrence of diabetic ketosis or ketoacidosis approximately 3 months after the onset of hyperglycaemic symptoms (polydipsia, polyuria, and weight loss), (2) need for continuous insulin therapy after diagnosis of T1DM, (3) the presence of positive ant-islet autoantibodies (islet cell autoantibodies, glutamic acid decarboxylase autoantibodies, insulinoma-associated antigen 2 autoantibodies, or insulin autoantibodies), and (4) presence of endogenous insulin deficiency without verifiable antiislet autoantibodies. Acute onset T1DM (autoimmune) was diagnosed with fulfilment of criteria 1,2, and 3. Acute onset T1DM was diagnosed with fulfilment of criteria 1,2 and $4 .{ }^{6}$ )

\section{Study design}

This retrospective study was performed between 2015-2020 at the Diabetes Clinic of the University Hospital of Athens (P \& A Kyriakou). Ethical approval was obtained from the University Committee of Research Ethics. The IRB Number was 293/1201-2015. Informed consent was obtained from patients/parents. We studied basic parameters that are associated with multiple autoimmunity.

\section{1) Present age}

Patients were categorized in 3 groups based on age (children $\leq 5$ years, children older than 5 but younger than 10 years, and children $>10$ years). Our main aim was to compare these groups according to severity of T1DM clinical presentation, number and titre of pancreatic autoantibodies at diabetes diagnosis (glutamic acid decarboxylase antibodies [GADA] and islet antigen 2 [IA2]), fasting C-peptide level, and the presence of familial autoimmunity. For study of multiple autoimmunity, the age categories were children $\leq 5$ years, children older than 5 but younger than 12 years, and children $>12$ years.

2) Severity of clinical and laboratory parameters at diagnosis

Diabetic ketoacidosis (DKA) severity was classified according to International Society for Pediatric and Adolescent Diabetes (ISPAD) criteria as mild ( $\mathrm{pH}$ venous blood $<7.3$ or $\mathrm{HCO}_{3}$ $<15 \mathrm{mmol} / \mathrm{L}$ ), moderate ( $\mathrm{pH}$ venous blood $<7.2$ or $\mathrm{HCO}_{3}$ $<10 \mathrm{mmol} / \mathrm{L}$ ), and severe ( $\mathrm{pH}$ venous blood $<7.1$ or $\mathrm{HCO}_{3}<5$ $\mathrm{mmol} / \mathrm{L})^{7)}$

3) Associated autoimmunity-multiple autoimmunity

We studied any associated autoimmune diseases diagnosed before or simultaneously with T1DM diagnosis or diagnosed during the follow-up periods. The diagnosis was made by consideration of the symptoms, treatment was instituted, and the presence of autoantibodies was assessed. These assessed autoantibodies included anti-thyroid (anti-TPO/anti-TG), celiac disease (anti-tTG-IgA/anti-EMA-IgA), and antiparietal cell antibodies (APCA).

Associated autoimmunity was defined as the presence of one or more autoimmune diseases in addition to T1DM. An additional autoimmune condition diagnosis was defined as double autoimmunity, 2 additional diagnoses were defined as triple autoimmunity, and 3 or more autoimmunity diagnoses resulted in quadruple autoimmunity. Triple and quadruple autoimmunity were defined as multiple autoimmunity.

Thyroid autoimmunity was diagnosed when thyroid autoantibodies (anti-TPO and/or anti-TG) were present and thyroid-stimulating hormone (TSH) and free T4 (FT4) levels were within the normal range. Subclinical autoimmune thyroiditis was diagnosed when one thyroid autoantibody was present on 2 or more consecutive measurements and/or thyroiditis was identified on ultrasound in conjunction with high TSH level, 6-9 $\mu \mathrm{IU} / \mathrm{L}$; normal free T3 and FT4 levels; and absence of symptoms. Clinical hypothyroidism was defined as the presence of thyroid autoantibodies in conjunction with high TSH, $\geq 10 \mu \mathrm{IU} / \mathrm{L}$; abnormal FT4 level and/or pathological findings on thyroid ultrasound; and presence of clinical symptoms.

The presence of autoantibodies against parietal cells of the stomach (APCA) was defined as gastric autoimmunity; however, the diagnosis of autoimmune gastritis required abnormal gastric biopsy findings. 
All patients at diagnosis and during yearly follow-up were tested for parathyroid hormone, serum calcium/ phosphate, and 25-hydroxyvitamin D3 levels. Moreover, the growth and pubertal maturation of patients were monitored during follow-up. In cases of pubertal delay, assessment of gonadal autoimmunity was indicated. We did not routinely investigate autoimmunity against adrenal glands and gonads as previous studies $^{8,9)}$ had shown that adrenal and gonadal autoimmunity are not present in childhood and adolescent T1DM.

However, patients who presented with unexplained hypoglycemia were investigated for Addison disease and CD according to ISPAD guidelines. ${ }^{10)}$ Patients were assessed for Addison disease if they presented with fatigue, weakness, gastrointestinal discomfort, salt craving, and hyperpigmentation or electrolyte abnormalities. A family history of Addison disease was another indication for this assessment.

\section{4) Familial autoimmunity}

Familial autoimmunity is the presence of at least one autoimmune disease within either the nuclear or extended, defined as second- and third-degree relatives, family.

\section{Measurements}

GADA and antibodies against tyrosine phosphatase (IA2) were measured with the radioimmunoassay method; the highest normal limit for these measurements was $0.9 \mathrm{U} / \mathrm{mL}$ and $0.75 \mathrm{U} /$ $\mathrm{mL}$, respectively. The normal range for fasting $\mathrm{C}$-peptide was $1.77-4.68 \mathrm{ng} / \mathrm{mL}$.

Antibodies against anti-TG and anti-TPO were determined using the luminescence method (ILMA, Nichols, Germany) and Advantage analyzer. Positive titres were considered $>100 \mathrm{IU} / \mathrm{mL}$ for anti-TG and $>16 \mathrm{IU} / \mathrm{mL}$ for anti-TPO.

Diagnosis of $\mathrm{CD}$ was based on antibodies against tissue transglutaminase (tTG-IgA) or against gliadin (AGA IgA); in cases of $\operatorname{Ig}$ A deficiency, determination of $\operatorname{IgG}$ antibodies was performed. In all cases, diagnosis was confirmed by jejunal biopsy, typical findings on which included lymphocytic infiltration and hypertrophy of the crypts and villous atrophy (Marsh II classification). Anti-tTG-IgA class antibodies were detected by the enzyme-linked immunosorbent assay method, and normal levels were considered to be 20 units. Weak positivity was diagnosed when levels were 20-30 units.

The presence of gastric autoimmunity was based on positive APCA. The determination of APCA was performed using the indirect immunofluorescent assay on a LEICA fluorescent microscope (DM LB2 Germany). Titres > 1:40 for APCA were positive.

The mean value of glycated hemoglobin (HbAlc) was deducted from all measurements performed during the last year of follow-up. The normal range was $4.6 \%-6.2 \%$.

\section{Statistical analysis}

All continuous variables are presented as mean value \pm standard deviation (SD) when normally distributed and as median value (interquartile range, IQR) when not. Normal distribution was assessed by Kolmogorov-Smirnov test. Chisquare test and Fisher exact test were used for categorical variables. Student $t$-test was used for variables normally distributed, and nonparametric Mann-Whitney $U$-test was used for variables not following normal distribution. Kruskal-Wallis test was used to compare quantitative and qualitative variables in more than 2 groups. For linear correlation, Spearman RHO test was performed. To assess the overall effect of the factors related to DKA probability, univariate logistic regression analysis was initially carried out. This was followed by multifactorial logistic regression analysis to evaluate adjusted odds ratio. Analysis was also performed by age group and categorized in 2 models: (1) $\leq 5$ years old, older than 5 years but younger than 10 years, $>10$ years old and $(2) \leq 2$ years old, older than 2 but younger than 12 old, and $>12$ years old. The data analysis was performed with the use of IBM SPSS Statistics ver. 21.0 (IBM Co., Armonk, NY, USA).

\section{Results}

\section{Demographic and laboratory characteristics}

From the 121 children included in the study, 63 were male (52.1\%). Mean age \pm SD was $11.99 \pm 4.63$ years (range, $2.0-20.0$ years), age at T1DM diagnosis was 7.62 $\$ 3.67$ years (range, 1.015.0 years), and median disease duration was 3.5 years (range, 2 months to 14.5 years). At T1DM diagnosis, mean $\mathrm{pH} \pm \mathrm{SD}$ was $7.21 \pm 0.15$ (range, 6.83-7.46), and median $\mathrm{HCO}_{3}$ was $9 \mathrm{mmol} /$ $\mathrm{L}$ (range, $0.90-29.0 \mathrm{mmol} / \mathrm{L}$ ). GADA median value at diagnosis was $4.57 \mathrm{U} / \mathrm{mL}$ and IA2 median value was $4.00 \mathrm{U} / \mathrm{mL}$. GADA was present in $73.5 \%$ of children, while IA 2 was present in $71.4 \%$. Mean \pm SD HbAlc level during the last year of follow-up was $7.66 \% \pm 1.16 \%$. Median C-peptide at T1DM diagnosis was $0.59 \mathrm{ng} / \mathrm{mL}$ (IQR, 0.45-0.86 ng/mL).

\section{Associated autoimmunity}

Associated autoimmunity was present in $28.9 \%$ of the patients, while familial autoimmunity within the nuclear and/ or the extended family occurred in $62.8 \%$. At diabetes diagnosis, 15 children (12.4\%) manifested associated autoimmunity, $14(11.6 \%)$ had double autoimmunity, and one had triple autoimmunity. During the follow-up period, 34 children (28.1\%) developed associated autoimmunity, 27 (22.3\%) of whom manifested double autoimmunity, 6 (5\%) of whom demonstrated triple autoimmunity, and $1(0.8 \%)$ had quadruple autoimmunity.

The frequency of associated autoimmunity was: thyroid autoimmunity/autoimmune Hashimoto thyroiditis (25.2\%), 
CD (7.1\%), and gastric autoimmunity (5.2\%). One child (3.4\%) had been diagnosed with autoimmune thyroiditis before T1DM diagnosis, 11 children (36.6\%) were simultaneously diagnosed with T1DM and thyroid autoimmunity, and 18 children (60.0\%) were diagnosed with autoimmune thyroiditis after T1DM diagnosis. Three children were simultaneously diagnosed with T1DM and CD (37.5\%), and 5 children (62.5\%) developed CD during diabetes follow-up. Only 1 child presented with autoimmune gastritis at the time of diabetes diagnosis (20\%) and $4(80 \%)$ were diagnosed later. Three children $(2.5 \%)$ presented with positive celiac antibodies (tTG-IgA/EMA-IgA) without histological confirmation of the disease.

\section{Associated autoimmunity-clinical and laboratory characteristics}

Of children with associated autoimmunity, $42.9 \%$ were males and $57.1 \%$ were females. At T1DM diagnosis, $33.3 \%$ of male and $66.7 \%$ of female patients were diagnosed with associated autoimmunity. Female sex was associated with higher frequency of associated autoimmunity (no statistical significance).

Children who presented with associated autoimmunity at diabetes diagnosis were older compared to those without (mean $9.90 \pm 3.85$ years vs. $7.29 \pm 3.55$ years, $P=0.009$ ). The presence of associated autoimmunity during diabetes follow-up was not affected by the age at T1DM diagnosis.

Children with associated autoimmunity were older than those without at the time of the study (mean of present age $13.59 \pm 3.93$ years and $11.47 \pm 4.77$ years, respectively, $P=0.014$ ), and had longer diabetes duration (median 4.50 years vs. 3.00 years, respectively, $P=0.044$ ) (Table 1 ).

Subsequently, T1DM children were divided into 3 age groups based on the age at diabetes diagnosis ( 1 to 5 , more than 5 through 12 , and $>12$ years) and the present age (Table 2 ). Patients who were older at diabetes diagnosis had higher prevalence of associated autoimmunity $(P=0.059)$. Adolescents who belonged to the 12-15 years age group had a 7 -fold higher chance of developing associated autoimmunity compared to children between 1 and 5 (odds ratio [OR], 6.85; 95\% confidence interval $[\mathrm{CI}], 1.25-37.60 ; P=0.027)$. At the time of this study, older children ( $>12$ years) were also more prone to develop associated autoimmunity $(P=0.015)$. Both at diagnosis and at the time of study, children $>12$ years were more likely to develop associated autoimmunity.

\section{Associated autoimmunity}

1) Thyroid autoimmunity

Thirty children developed thyroid autoimmunity during the follow-up period, of whom 14 were male (46.7\%) and 16 female

Table 1. Association between the present age and diabetes duration with the presence of associated autoimmunity during the follow-up

\begin{tabular}{lccc}
\hline & Present age (yr) & $P$-value & Diabetes duration (yr) \\
\hline Associated autoimmunity during follow-up & & 0.014 & \\
Yes & $13.59 \pm 3.93$ & & $4.50(2.50-8.0)$ \\
No & $11.47 \pm 4.77$ & & $3.00(1.00-6.0)$ \\
\hline
\end{tabular}

Values are presented as mean \pm standard deviation or median (interquartile range).

Table 2. Associated autoimmunity at T1DM diagnosis and at the present age based on age category

\begin{tabular}{|c|c|c|c|c|c|}
\hline T1DM diagnosis age category & $1-5$ Years & $5.1-12$ Years & $12.1-15$ Years & $12.1-20$ years & $P$-value \\
\hline AA at diabetes diagnosis & & & & & 0.059 \\
\hline Yes & $3(7.7)$ & $8(11.3)$ & $4(36.4)$ & - & \\
\hline No & $36(92.3)$ & $63(88.7)$ & $7(63.6)$ & - & \\
\hline AA at present age category & & & & & 0.015 \\
\hline Yes & $1(9.1)$ & $9(18.8)$ & - & $25(40.3)$ & \\
\hline No & $10(90.9)$ & $39(81.3)$ & - & $37(59.7)$ & \\
\hline
\end{tabular}

Values are presented as number (\%).

TIDM, type 1 diabetes mellitus; AA, associated autoimmunity.

Table 3. Correlations of different types of associated autoimmunity in T1DM children with various parameters.

\begin{tabular}{|c|c|c|c|c|c|c|}
\hline \multirow{2}{*}{$\begin{array}{l}\text { Variable } \\
\text { Autoimmunity presence }\end{array}$} & \multicolumn{2}{|c|}{ Thyroid autoimmunity } & \multicolumn{2}{|c|}{ Celiac disease } & \multicolumn{2}{|c|}{ Gastric autoimmunity } \\
\hline & Yes & No & Yes & No & Yes & No \\
\hline Sex, male:female & $14: 16$ & $48: 41$ & $2: 6$ & $56: 49$ & $2: 3$ & $47: 44$ \\
\hline Present age (yr) & $13.62 \pm 4.17^{*}$ & $11.66 \pm 4.65^{*}$ & $12.75 \pm 3.06$ & $11.97 \pm 4.74$ & $13.90 \pm 2.90$ & $12.40 \pm 4.70$ \\
\hline Age at T1DM diagnosis (yr) & $7.68 \pm 3.90$ & $7.64 \pm 3.58$ & $7.63 \pm 4.53$ & $7.61 \pm 3.65$ & $10.20 \pm 3.09$ & $7.53 \pm 3.70$ \\
\hline Age at disease diagnosis (yr) & $9.70 \pm 3.83$ & - & $9.31 \pm 3.75$ & - & $11.60 \pm 2.63$ & - \\
\hline Symptomatic disease & $7 / 30(23.3)$ & - & $3 / 8(37.5)$ & - & - & - \\
\hline
\end{tabular}

Values are presented as number (\%).

T1DM, type 1 diabetes mellitus.

${ }^{*} P<0.05$, statistically significant differences. 
(53.3\%). The mean \pm SD age of thyroid autoimmunity diagnosis was $9.70 \pm 3.83$ years, and mean age at diabetes diagnosis in children who developed thyroid autoimmunity was $7.68 \pm 3.90$ years. This was not significantly different from that of children without thyroid autoimmunity $(7.64 \pm 3.58$ years). T1DM children with thyroid autoimmunity were older compared to their counterparts without (mean age: $13.62 \pm 4.17$ years and $11.66 \pm 4.65$ years, $P=0.043$ ) (Table 3 ). Among children with thyroid autoimmunity, seven of $30(23.3 \%$ or $5.8 \%$ of the total study population) eventually developed clinical disease and were treated with thyroxin. No association was found between clinical disease and sex or presence of positive GADA at diabetes diagnosis.

\section{2) Celiac disease}

Of the 8 children who developed celiac disase, 2 were male $(25.0 \%)$ and 6 female (75.0\%). Mean age at CD diagnosis was $9.31 \pm 3.75$ years. The mean age at T1DM diagnosis for children with CD was $7.63 \pm 4.53$ years, and the mean \pm SD present age was $12.75 \pm 3.06$ years. The mean \pm SD age at diabetes diagnosis for children without $\mathrm{CD}$ was $7.61 \pm 3.65$ years, and mean \pm SD current age was $11.97 \pm 4.74$ years. These were not statistically significantly different. From children with CD, 5 (62.5\%) were asymptomatic and diagnosis was confirmed

Table 4. The prevalence of associated autoimmune diseases according to different age groups

\begin{tabular}{lcccc}
\hline Present age group & $1-5$ years & $5.1-12$ years & $12.1-20$ years & $P$-value \\
\hline Thyroid autoimmunity & & & 0.019 \\
Yes & $1(10.0)$ & $7(14.6)$ & $22(36.1)$ & \\
No & $9(90.0)$ & $41(85.4)$ & $39(63.9)$ & \\
Celiac disease & & & & 0.763 \\
Yes & $0(0)$ & $4(8.9)$ & $4(7.0)$ & \\
No & $11(100)$ & $41(91.1)$ & $53(93.0)$ & \\
Gastric autoimmunity & & & & 0.786 \\
Yes & $0(0)$ & $1(3.0)$ & $4(7.4)$ & \\
No & $9(100)$ & $32(97.0)$ & $50(92.6)$ & \\
\hline
\end{tabular}

Values are presented as number (\%). with presymptomatic screening; 3 (37.5\%) developed clinical manifestations such as bloating, abdominal pain and irondeficiency anaemia (Table 3).

\section{3) Gastric autoimmunity}

Gastric autoimmunity prevalence during T1DM followup was $40.0 \%$ for male and $60.0 \%$ for female patients. The mean \pm SD age at diagnosis was $11.60 \pm 2.63$ years. Children who developed gastric autoimmunity were older at diabetes diagnosis (mean age, $10.20 \pm 3.09$ years) compared to those without (mean age, $7.53 \pm 3.70$ years). This difference was not statistically significant. Four were asymptomatic, and 1 manifested symptoms attributed to Helicobacter pylori infection (Table 3). None of our patients developed clinical symptoms or laboratory findings of hypoparathyroidism, hypogonadism or Addison's disease.

\section{Age and autoimmunity}

The majority of children with thyroid autoimmunity was diagnosed at the age of $5-12$ years $(53.3 \%)$ and $12-15$ years $(30 \%)$; only $16.7 \%$ of those 5 years or below developed the disease (Table 4). Gastric autoimmunity presented at older ages ( 5 children were $>5$ years and none were younger). Two children with CD were diagnosed at age less than 5 years, 5 children at age between 5 through 12 years, and 1 child older than 12 years.

Children between 12 and 20 years had a statistically significantly higher chance of developing thyroid autoimmunity compared to those 1 through 5 years old $(P=0.019)$. Despite not being statistically significant, the frequency of gastric autoimmunity increased in parallel with increasing present age, but the frequency of CD decreased after the age of 12 years (Table 4).

Subsequently, multivariate analysis was performed with sex added as a confounding factor. Children older than 12 years had 3-fold higher chance of developing thyroid autoimmunity, irrespective of sex (OR, 3.23; 95\% CI, 1.25-8.32; $P=0.015)$. No statistically significant difference was found for CD and gastric

Table 5. Association between antipancreatic (GADA and IA2) antibodies and the presence of associated autoantibodies (thyroid, coeliac, APCA) in children with newly diagnosed T1DM

\begin{tabular}{|c|c|c|c|c|}
\hline Variable & GADA (U/mL) & $P$-value & $\mathrm{IA} 2(\mathrm{U} / \mathrm{mL})$ & $P$-value \\
\hline Anti-TPO/anti-TG & & 0.026 & & 0.414 \\
\hline Positive & $10.26(1.45-38.59)$ & & $3.00(0.60-10.79)$ & \\
\hline Negative & $3.88(0.56-10.93)$ & & 5.00 (0.74-12.99) & \\
\hline tTGlgA/EMA-lgA & & 0.377 & & 0.528 \\
\hline Positive & $0.90(0.40-27.00)$ & & $2.03(0.50-11.00)$ & \\
\hline Negative & $4.87(1.00-21.47)$ & & $4.60(0.70-12.46)$ & \\
\hline APCA & & 0.030 & & 0.946 \\
\hline Positive & $29.94(24.18-35.73)$ & & $11.00(0.10-15.00)$ & \\
\hline Negative & $4.16(0.80-10.26)$ & & $4.30(0.74-12.99)$ & \\
\hline
\end{tabular}

Values are presented as median (interquartile range).

GADA, glutamic acid decarboxylase autoantibodies; IA2, protein tyrosine phosphatase-like protein IA2; APCA, antibodies against parietal cells; anti-TPO/anti-TG, antibodies against thyroid peroxidase/thyroglobulin; tTG-IgA/EMA-IgA, IgA antibodies against tissue transglutaminase/endomysial. 
Table 6. Characteristics of children with T1DM and multiple autoimmunity compared to their counterparts with one or no additional autoimmune disease

\begin{tabular}{|c|c|c|c|c|c|}
\hline Characteristic & T1DM and $\geq 2$ & T1DM + 1 autoimmune & No autoimmune disease & $P$-value ${ }^{\dagger}$ & $P$-value ${ }^{\ddagger}$ \\
\hline Present age (yr) & $13.36 \pm 3.20$ & $13.64 \pm 4.14$ & $11.47 \pm 4.77$ & 0.093 & 0.309 \\
\hline Sex, male:female & $3: 4(42.9: 57.1)$ & $12: 16(42.9: 57.1)$ & $48: 38(55.8: 44.2)$ & 0.435 & 0.697 \\
\hline Age at diabetes diagnosis (yr) & $7.57 \pm 4.73$ & $8.05 \pm 3.70$ & $7.48 \pm 3.61$ & 1.000 & 0.948 \\
\hline Age at diabetes diagnosis $<5$ years, yes:no & $3 / 4(42.9 / 57.1)$ & $8 / 20(28.6 / 71.4)$ & $28 / 58(32.6 / 67.4)$ & 0.789 & 0.682 \\
\hline T1DM duration (age) & $4.0(1.5-11.0)$ & $4.75(2.5-7.5)$ & $3.0(1.0-6.0)$ & 0.132 & 0.277 \\
\hline Mean HbA1c (last year) & $7.41 \pm 0.79$ & $7.82 \pm 1.35$ & $7.63 \pm 1.13$ & 1.000 & 0.605 \\
\hline 2 Antipancreatic antibodies, yes:no & $1: 3(20.0: 80.0)$ & $12: 2(85.7: 14.3)$ & $37: 7(84.1: 15.9)$ & 1.000 & 1.000 \\
\hline DKA at diagnosis, present:absent & $6: 0(100: 0)$ & $15: 10(60.0: 40.0)$ & $57: 28(67.1: 32.7)$ & 0.190 & 0.172 \\
\hline Moderate/severe DKA at diagnosis, present:absent & $4: 2(66.7: 33.3)$ & $8: 17(32.0: 68.0)$ & $47: 38(55.3: 44.7)$ & 0.085 & 0.691 \\
\hline Familial autoimmunity, yes:no & $7: 0(100: 0.0)$ & $18: 10(64.3: 35.7)$ & $51: 35(59.3: 40.7)$ & 0.080 & 0.042 \\
\hline No. of relatives with familial autoimmunity $\geq 3$, yes:no & $2: 5(28.6: 71.4)$ & $8: 20(28.6: 71.4)$ & $8: 78(9.3: 90.7)$ & 0.026 & 0.163 \\
\hline
\end{tabular}

Values are presented as mean \pm standard deviation, number (\%), or median (interquartile range).

T1DM, type 1 diabetes mellitus; HbA1c, hemoglobin A1c; DKA, diabetic ketoacidosis.

${ }^{\dagger}$ Comparison of all 3 groups. ${ }^{\ddagger}$ Comparison of T1DM group and group with $\geq 2$ autoimmune diseases versus group with no associated autoimmune diseases.

autoimmunity.

Children who developed thyroid autoimmunity, CD, or gastric autoimmunity did not have statistically significantly higher frequency or severity of DKA at diabetes diagnosis compared to children without autoimmunity.

\section{Associated autoimmunity and pancreatic autoimmunity}

GADA titre at diabetes diagnosis was significantly higher in children with positive thyroid autoantibodies and APCA ( $P=0.026$, kaı 0.030 , respectively, Mann-Whitney Test). There was no association between GADA or IA2 and coeliac antibody presence (Table 5).

\section{Characteristics of patients with multiple autoimmunity}

Multiple autoimmunity was present in $5.8 \%$ of the study population. Children with multiple autoimmunity manifested moderate to severe DKA more frequently at T1DM diagnosis (not statistically significant). Nevertheless, all children with multiple autoimmunity had developed DKA at T1DM diagnosis. Children who had $\geq 2$ autoimmune diseases were older $(P=0.093)$ and were diagnosed at an age younger than 5 years compared to their counterparts with no associated autoimmunity (Table 6).

Children with multiple autoimmunity had a higher percentage of positive familial history of autoimmunity compared to children without $(P=0.042)$. Children with multiple autoimmunity more frequently had $\geq 3$ relatives with autoimmunity compared to those without $(P=0.026)$ (Table 6 ). There was no statistically significant difference between children with or without multiple autoimmunity in terms of sex, age at diabetes diagnosis, T1DM duration or the presence of 2 antipancreatic autoantibodies.
1) Multiple logistic regression analysis

The presence and number of associated autoimmune diseases were not related to the age at diabetes diagnosis $(P=0.948)$ or to the presence of DKA at diabetes diagnosis $(P=0.152)$ (Table 6).

Multiple logistic regression analysis was performed (Supplementary Table 1) to investigate the effect of current age and T1DM duration on associated autoimmunity. In this analysis, the presence of associated autoimmunity was the dependent variable and current age, age at diabetes diagnosis, diabetes duration, sex, and familial history of autoimmunity were independent variables. When diabetes duration was excluded from the equation, current age was the most significant variable affecting associated autoimmunity development $(P=0.010)$. When excluding current age, diabetes duration was the most significant variable $(P=0.011)$. However, when including both current age and diabetes duration, none of the remaining variables significantly affected associated autoimmunity. Therefore, current age and T1DM duration equally affect associated autoimmunity development in children and adolescents with T1DM.

\section{Discussion}

The present study reports on the prevalence of associated and multiple autoimmunity in children and adolescents with T1DM. Among the novel findings of our study are the identification of multiple autoimmunity risk factors and their association with familial autoimmunity. To our knowledge previous similar studies are rare.

Associated autoimmunity was detected in $28.9 \%$ of the study population, with $23.1 \%$ having double autoimmunity, $5 \%$ triple, and $0.8 \%$ quadruple autoimmunity. Multiple autoimmunity ( $\geq 2$ autoimmune diseases) was found in $5.8 \%$ of our study population. Previous studies have reported that the prevalence of associated autoimmunity in children and adolescents with T1DM fluctuates between 27\%-34\%. ${ }^{11,12)}$ Multiple 
autoimmunity was not investigated in any previous studies.

The most frequently encountered associated autoimmune disease in this study was thyroid autoimmunity, which presented in $25.2 \%$ children and adolescents. Thyroid autoimmunity prevalence in children with T1DM has been reported to be between $12.1 \%$ and $23.4 \%{ }^{13,14)}$ In our study, thyroid autoimmunity was positively associated with older age and longer diabetes duration. This finding agrees with those of previous studies. ${ }^{12,15,16)}$ However, when comparing the effects of older age and diabetes duration on associated autoimmunity development, multiple logistic regression analysis demonstrated that both variables were equally significant factors when excluding the effects of the other in the equation. We have also shown that the highest prevalence of thyroid antibody positivity was observed around puberty (14-15 years) and after $3.5-4$ years of diabetes duration. The increased prevalence of thyroid autoimmunity in adolescence is possibly related to the effect of oestrogens. ${ }^{17)}$ In animal models, oestrogens accelerate the evolution of autoimmune diseases. ${ }^{18)}$ Clinical or subclinical hypothyroidism treated with levothyroxine was seen in $58 \%$ of the total study population. There was no association between clinical or subclinical hypothyroidism treated with levothyroxine and GADA antibody positivity. Prognostic factors for thyroxine administration among children diagnosed with T1DM before 5 years of age were female and positive for GADA. Older children were anti-TPO positive with abnormal TSH level. ${ }^{19)}$

In this study $7.1 \%$ of T1DM patients were diagnosed with CD. The prevalence of CD in children with T1DM fluctuates between $1 \%-16 \%{ }^{20-22)}$ In our study, only $62.5 \%$ of children with autoantibodies against CD were symptomatic, which agrees with other studies. ${ }^{23,24)}$ The presence of autoantibodies against CD has been previously associated with younger age at diabetes diagnosis, shorter diabetes duration, and female sex. ${ }^{25,26)}$ In the present study, no significant association with the above parameters was found, possibly due to the small number of patients.

Gastric autoimmunity was found in $5.2 \%$ of the study population. Kakleas et al. ${ }^{27)}$ have reported positive APCA at diagnosis in $7.2 \%$ of children. The prevalence of gastric autoimmunity among T1DM children and adolescents in the literature has been reported to be $5.3 \%-5.6 \%{ }^{28)}$ with higher prevalence in young adults $(18 \%-20 \%){ }^{29)}$ Only one patient in our study had gastritis associated with Helicobacter pylori (HP) presence. The presence of HP is associated with autoimmune gastritis and is a marker of gastric atrophy. ${ }^{30)}$ In the present study, gastric autoimmunity prevalence increased with age. Though most studies report that APCA positivity is associated with older age, longer diabetes duration, ${ }^{31)}$ and female sex, ${ }^{32)}$ others demonstrated no association. This can be attributed to population or research methodology differences.

GADA titre was associated in a marginally nonstatistically significant manner with the presence of associated autoimmunity at diabetes diagnosis but not during the follow-up. Patients with positive thyroid autoantibodies and APCA were more likely to have high GADA titre. Previous studies have reported that GADA positivity persistence after 5 years from T1DM diagnosis and predisposes the individual to development of autoimmune thyroiditis and gastritis. ${ }^{33)}$ The relationship between GADA positivity and thyro-gastric antibody positivity in patients with T1DM can be explained by the common antigens of the pancreas, thyroid gland, and stomach. ${ }^{34)}$ As a result, once a $\mathrm{T}$-cell response to GAD has been primed in the pancreas, the resulting activated T cells can then initiate damage of other tissues containing similar enzyme(s). Thus, T1DM patients with high GADA titre at diabetes diagnosis or with persistence of GADA should be closely monitored for the development of thyroid and/or gastric autoimmunity.

Children with multiple autoimmunity all presented with DKA at T1DM diagnosis, were diagnosed at a younger age $(\leq 5$ years), and had higher prevalence of familial autoimmunity in multiple relatives ( $\geq 3$ relatives). The association of DKA at T1DM diagnosis and future development of multiple autoimmunity has been attributed to either a more aggressive T1DM endotype or the effect of DKA on the immune system. ${ }^{35)}$ In agreement with our results, previous studies have shown that multiple autoimmunity is associated with younger age at diabetes diagnosis, ${ }^{36,37)}$ higher frequency of DKA, ${ }^{31)}$ and increased frequency of complications and mortality. ${ }^{38)}$ Hence, T1DM diagnosis at a young age with a severe clinical presentation and the presence of a significant family history of autoimmunity could be used as predictors for multiple autoimmunity development.

None of our patients developed autoimmune hypoparathyroidism, hypogonadism, or Addison disease as part of autoimmune polyglandular syndromes types $1-3{ }^{39)}$ We recommend that all children and adolescents with T1DM be investigated at diagnosis for the presence of thyroid and gastric autoimmunity and CD. These children should be screened yearly thereafter for thyroid autoimmunity. Cases of GADA positivity persistence or symptoms of gastritis should also be screened for gastric autoimmunity. As the frequency of CD decreased after the age of 12 years, we suggest that patients should be screened annually for CD until the age of 12 years and biannually thereafter. Adrenal autoimmunity should be screened in T1DM patients aged at least 18 years, especially those in those with a family history of Addison disease. ${ }^{17)}$ However, with symptoms and signs of CD, autoimmune gastritis, Addison disease, or other autoimmune diseases as part of APS types 1-3, patients should be screened, regardless of negative previous screening, especially in the presence of a family history.

Limitations of this study are the relatively small number of participants and the higher percentage of recently diagnosed T1DM patients with shorter disease duration. This could prevent the development of statistically significant associations for specific parameters. Another limitation is the study's retrospective design. Nevertheless, the study participants, by originating at a tertiary hospital centre, are representative of the Greek population.

The present study is one of a few to investigate the effect of 
multiple autoimmunity on diabetes phenotype and clinical presentation. Furthermore, this study sought associations between the most basic autoimmunity-associated parameters and clinical and laboratory manifestations at diabetes diagnosis. These have not been extensively studied in children. Through the recognition of these potential risk factors, follow-up of patients with T1DM can be more focused. Another advantage of this study is the repeated annual testing of organ-specific autoantibodies as a part of the presymptomatic control. Therefore, diagnosis of the associated autoimmune diseases was performed both during the asymptomatic and clinical stages.

In conclusion, approximately one-third of children and adolescents with T1DM had associated autoimmunity and $5.8 \%$ had multiple autoimmunity. Predictors of associated autoimmunity were older age, longer diabetes duration, and female sex. Children with multiple autoimmunity were diagnosed with T1DM at an age younger than 5 years, and all had DKA at diabetes diagnosis and a significant family history of autoimmunity. As delayed diagnosis of associated and multiple autoimmunity is related to significant morbidity and mortality, regular screening of children and adolescents with T1DM for associated autoimmunity, especially those with GADA positivity and a significant family history of autoimmunity, is important for early identification and management of associated autoimmune diseases.

\section{Ethical statement}

Ethical approval was obtained from the University Committee of Research Ethics (IRB No. 293/12-01-2015). Informed consent was obtained from patients/parents.

\section{Notes}

Supplementary material: Supplementary Table 1 can be found via https://doi.org/10.6065/apem.2142168.084.

Conflicts of interest: No potential conflict of interest relevant to this article was reported.

Funding: This study received no specific grant from any funding agency in the public, commercial, or not-for-profit sectors.

Data availability: The data that support the findings of this study can be provided by the corresponding author upon reasonable request.

Author contribution: Conceptualization: KKakleas, KKaravanaki; Data curation: AK; Formal analysis: LK, NK; Methodology: LK, AK, KKaravanaki; Project administration: KKakleas, LK; Writing - original draft: KKakleas, AK, NK, SK; Writing - review \& editing: KKakleas, KKaravanaki

\section{ORCID}

Konstantinos Kakleas: 0000-0002-7993-8418

\section{References}

1. Mayer-Davis EJ, Kahkoska AR, Jefferies C, Dabelea D, Balde N, Gong CX, et al. ISPAD clinical practice consensus guidelines 2018: definition, epidemiology, and classification of diabetes in children and adolescents. Pediatr Diabetes 2018;19 Suppl 27(Suppl 27):7-19.

2. Barker JM. Clinical review: type 1 diabetes-associated autoimmunity: natural history, genetic associations, and screening. J Clin Endocrinol Metab 2006;91:1210-7.

3. Husebye ES, Anderson MS, Kämpe O. Autoimmune polyendocrine syndromes. N Engl J Med 2018;378:113241.

4. Kordonouri O, Klingensmith G, Knip M, Holl RW, Aanstoot HJ, Menon PS, et al. International Society for Pediatric and Adolescent Diabetes. ISPAD Clinical Practice Consensus Guidelines 2014. Other complications and diabetes-associated conditions in children and adolescents. Pediatr Diabetes 2014;15 Suppl 20:270-8.

5. Wägner AM, Santana A, Herńndez M, Wiebe JC, Nóvoa J, Mauricio D. Predictors of associated autoimmune diseases in families with type 1 diabetes: results from the Type 1 Diabetes Genetics Consortium. Diabetes Metab Res Rev 2011;27:493-8.

6. Kawasaki E, Maruyama T, Imagawa A, Awata T, Ikegami H, Uchigata Y, et al. Diagnostic criteria for acute-onset type 1 diabetes mellitus (2012): report of the Committee of Japan Diabetes Society on the Research of Fulminant and Acute-onset Type 1 Diabetes Mellitus. J Diabetes Investig 2014;5:115-8.

7. Wolfsdorf JI, Glaser N, Agus M, Fritsch M, Hanas R, Rewers A, et al. ISPAD Clinical practice consensus guidelines 2018: diabetic ketoacidosis and the hyperglycemic hyperosmolar state. Pediatr Diabetes 2018;19 Suppl 27:155-77.

8. Michels AW, Gottlieb PA. Autoimmune polyglandular syndromes. Nat Rev Endocrinol 2010;6:270-7.

9. De Block CE, de LeeuwIH, Vertommen JJ, Rooman RP, Du CajuMV, Van Campehout CM, et al. Beta-cell, thyroid, gastric, adrenal and coeliac autoimmunity and HLA-DQ types in type 1 diabetes. Clin Exp Immunol 2001;126:23641.

10. Abraham MB, Jones TW, Naranjo D, Karges B, Oduwole A, Rauschmann M, et al. ISPAD clinical practice consensus guidelines 2018: assessment and management of hypoglycemia in children and adolescents with diabetes. Pediatric Diabetes 2018;19 Suppl 27:178-92.

11. Hughes JW, Riddlesworth TD, DiMeglio LA, Miller KM, Rickels MR, McGill JB, et al. Autoimmune diseases in children and adults with type 1 diabetes from the T1D exchange clinic registry. J Clin Endocrinol Metab 2016;101:4931-7.

12. Głowińska-Olszewska B, Michalak J, Łuczyński W, Del Pilar Larosa M, Chen S, Furmaniak J, et al. Organ-specific autoimmunity in relation to clinical characteristics in children with long-lasting type 1 diabetes. J Pediatr 
Endocrinol Metab 2016;29:647-56.

13. Jung ES, Han DK, Yang EM, Kim MS, Lee DY, Kim CJ. Thyroid autoimmunity in children and adolescents with newly diagnosed type 1 diabetes mellitus. Ann Pediatr Endocrinol Metab 2014;19:76-9.

14. Hwang GB, Yoon JS, Park KJ, Lee HS, Hwang JS. Prevalence of autoimmune thyroiditis in patients with type 1 diabetes: a long-term follow-up study. Ann Pediatr Endocrinol Metab 2018;23:33-7.

15. Kakleas K, Paschali E, Kefalas N, Fotinou A, Kanariou M, Karayianni C, et al. Factors for thyroid autoimmunity in children and adolescents with type 1 diabetes mellitus. Ups J Med Sci 2009;114:214-20.

16. Borzutzky A, Seiltgens C, Iruretagoyena M, Cristi F, Ponce MJ, Melendez P, et al. A126: clusters of autoimmune diseases in children and the role of PTPN22 C1858T Gene polymorphism in pediatric polyautoimmunity. Arthritis Rheumatol 2014;66:S164-5.

17. Kakleas K, Soldatou A, Karachaliou F, Karavanaki K. Associated autoimmune diseases in children and adolescents with type 1 diabetes mellitus (T1DM). Autoimmun Rev 2015;14:781-97.

18. Taneja V. Sex hormones determine immune response. Front Immunol 2018;9:1931.

19. Jonsdottir B, Larsson C, Carlsson A, Forsander G, Ivarsson SA, Lernmark $\AA$, et al. Thyroid and islet autoantibodies predict autoimmune thyroid disease at type 1 diabetes diagnosis. J Clin Endocrinol Metab 2017;102:1277-85.

20. Sahin Y. Celiac disease in children: a review of the literature. World J Clin Pediatr 2021;10:53-71.

21. Kylökäs A, Kaukinen K, Huhtala H, Collin P, Mäki M, Kurppa K. Type 1 and type 2 diabetes in celiac disease: prevalence and effect on clinical and histological presentation. BMC Gastroenterol 2016;16:76.

22. Salardi S, Volta U, Zucchini S, Fiorini E, Maltoni G, Vaira $\mathrm{B}$, et al. Prevalence of celiac disease in children with type 1 diabetes mellitus increased in the mid-1990s: an 18-year longitudinal study based on anti-endomysial antibodies. J Pediatr Gastroenterol Nutr 2008;46:612-4.

23. Husby S, Koletzko S, Korponay-Szabo IR, Mearin ML, Phillips A, Shamir R, et al. European Society for Pediatric Gastroenterology, Hepatology, and Nutrition guidelines for the diagnosis of coeliac disease. J Pediatr Gastroenterol Nutr 2012;54:136-60.

24. Ludvigsson JF, Neovius M, Hammarström L. Association between IgA deficiency \& other autoimmune conditions: a population-based matched cohort study. J Clin Immunol 2014;34:444-51.

25. Kakleas K, Karayianni C, Critselis E, Papathanasiou A, Petrou V, Fotinou A, et al. The prevalence and risk factors for coeliac disease among children and adolescents with type 1 diabetes mellitus. Diabetes Res Clin Pract 2010;90:202-8

26. Cerruti F, Bruno G, Chiarelli F, Lorini R, Meschi F, Sacchetti C. Younger age at onset and sex predict coeliac disease in children and adolescents with type 1 diabetes. Diabetes Care 2004;27:1294-8.

27. Kakleas K, Kostaki M, Critselis E, Karayianni C, Giannaki M, Anyfantakis K, et al. Gastric autoimmunity in children and adolescents with type 1 diabetes: a prospective study. Horm Res Paediatr 2012;77:121-6

28. Frohlich-Reiterer EE, Huber J, Katz H, Suppan E, Obermayer-Pietsch B, Deutschmann A, et al. Do children and adolescents with type 1 diabetes mellitus have a higher frequency of parietal cell antibodies than healthy controls? J PGN 2011;52:558-62.

29. De Block CE, De Leeuw IH, Van Gaal LF. High prevalence of manifestations of gastric autoimmunity in parietal cell antibody-positive type 1 (insulin-dependent) diabetic patients. The Belgian Diabetes Registry. J Clin Endocrinol Metab 1999;84:4062-7.

30. Conti L, Annibale B, Lahner E. Autoimmune gastritis and gastric microbiota. Microorganisms 2020;8:1827.

31. Fröhlich-Reiterer EE, Huber J, Katz H, Suppan E, Obermayer-Pietsch B, Deutschmann A, et al. Do children and adolescents with type 1 diabetes mellitus have a higher frequency of parietal cell antibodies than healthy controls? J Pediatr Gastroenterol Nutr 2011;52:558-62.

32. De Block CE, De Leeuw IH, Bogers JJ, Pelckmans PA, Ieven MM, Van Marck EA, et al. Helicobacter pylori, parietal cell antibodies and autoimmune gastropathy in type 1 diabetes mellitus. Aliment Pharmacol Ther 2002;16:281-9.

33. Karavanaki K, Kakleas K, Paschali E, Kefalas N, Konstantopoulos I, Petrou V, et al. Screening for associated autoimmunity in children and adolescents with type 1 diabetes mellitus (T1DM). Horm Res 2009;71:201-6.

34. Esteban M, Baxter AG. Polyspecificity of autoimmune responses in type 1 (autoimmune) diabetes. Clin Exp Immunol 2001;126:184-6.

35. Kakleas K, Basatemur E, Karavanaki K. Association between severity of diabetic ketoacidosis at diagnosis and multiple autoimmunity in children with type 1 diabetes mellitus: a study from a Greek Tertiary Centre. Can J Diabetes 2021;45:33-8.e2.

36. Krzewska A, Ben-Skowronek I. Effect of associated autoimmune diseases on type 1 diabetes mellitus incidence and metabolic control in children and adolescents. Biomed Res Int 2016;2016:6219730.

37. Kordonouri O, Maguire AM, Knip M, Schober E, Lorini R, Holl RW, et al. Other complications and associated conditions with diabetes in children and adolescents. Pediatr Diabetes 2009;10 Suppl 12:204-10.

38. Rogers MAM, Wei MY, Kim C, Lee JM. Sex differences in autoimmune multimorbidity in type 1 diabetes mellitus and the risk of cardiovascular and renal disease: a longitudinal study in the United States, 2001-2017. J Womens Health (Larchmt) 2020;29:511-9.

39. Eisenbarth GS, Gottlieb PA. Autoimmune polyendocrine syndromes. N Engl J Med 2004;350:2068-79. 